\title{
Some Implications of Liapunov's Conditions for Stability
}

\section{H. A. ANTOSIEWICZ*}

American University, Washington, D. C.

8

P. DAVIS

National Bureau of Standards, Washington, D. C.

Communicated by N. Levinson

1. Introduction. A. M. Liapunov [9] established several criteria for the stability of systems of ordinary differential equations which are independent of considerations of variational equations, and which form the basis of what Liapunov himself called the "second method." This method gave rise to a development of the theory of stability, to a large extent due to I. G. Malkin [10-16] and K. Persidskii [21-23] and numerous other Russian mathematicians, which has come into prominence anew in recent years.

In this paper we point out some implications of this theory of stability concerning linear as well as certain non-linear systems of differential equations and bring together more closely a number of related results which seem to have escaped attention thus far. In particular, we establish for the general linear system the equivalence of the existence of what is commonly called a Liapunov function with certain conditions upon any set of $n$ linearly independent solutions. This not only demonstrates the strong type of stability that is implied by the existence of such a Liapunov function, but also represents the connecting link between

* This work was performed under a National Bureau of Standards contract with The American University. 
this theory of stability and the more direct approach to stability criteria by means of the well known classical representation of the general solution of a linear system, as used by Perron [19, 20], Bellman [2, 3, 4], Levinson [7, 8] and others $[4,26]$. From a practical point of view, the equivalence of these two techniques is of importance because stability criteria based upon properties of the general solution of a linear system are oftentimes rather inaccessible while those based upon the existence of a Liapunov function are frequently easily applicable.

2. Liapunov's theorems on stability. For the sake of completeness we first recall a number of basic definitions and then state explicitly Liapunov's two theorems on stability.

We shall consider systems of differential equations of the form

$$
\frac{d x}{d t}=p(x, t)
$$

where $p(x, t)$ is a vector function of the vector $x$ and the (real) scalar variable $t$ which is continuous in $(x, t)$ and satisfies in $x$ a Lipschitz condition for every $(x, t)$ in some region $\Re:\|x\| \leqq A, t \geqq 0$. By $\|x\|$ we shall mean some convenient norm such as the Euclidean length of $x$. Then it is clear that given any vector $x_{0}$ and any scalar $t_{0} \geqq 0$ there exists a unique solution $x\left(t, x_{0}, t_{0}\right)$ of (1) which reduces to $x_{0}$ when $t=t_{0}$. Throughout we shall assume that $p(0, t) \equiv 0$ so that $x(t) \equiv 0$ is a (trivial) solution of (1).

The solution $x(t) \equiv 0$ is said to be stable if given any $t_{0} \geqq 0$ and $\epsilon>0$ there exist constants $\eta\left(\epsilon, t_{0}\right)>0$ and $T\left(\epsilon, t_{0}\right)>0$ such that every solution $x\left(t, x_{0}, t_{0}\right)$ of (1) satisfies $\left\|x\left(t, x_{0}, t_{0}\right)\right\| \leqq \epsilon$ for all $t \geqq T$ whenever $\left\|x_{0}\right\| \leqq \eta$.

The solution $x(t) \equiv 0$ is said to be asymptotically stable if it is stable and if there exists a fixed $\eta_{0}>0$ such that every solution $x\left(t, x_{0}, t_{0}\right) \rightarrow 0$ as $t \rightarrow \infty$ whenever $\left\|x_{0}\right\| \leqq \eta_{0}$.

We shall need throughout the following definitions due to Liapunov [9].

A scalar function $W(x)$, continuous for all $x$ in $\|x\| \leqq A$, is said to be positive definite if $W(x)>0$ for $x \neq 0$ and $W(0)=0$. A scalar function $V(x, t)$, continuous for all $(x, t)$ in $\Re$, is said to be positive definite if there exists a positive definite function $W(x)$ such that $V(x, t) \geqq W(x)$ for all $(x, t)$ in $\Re$ and $V(0, t) \equiv 0$; similarly for negative definite. A function $V(x, t)$ is said to admit an infinitely small upper bound if given any $\epsilon>0$ there exists a $\delta(\epsilon)>0$ such that $|V(x, t)| \leqq$ $\epsilon$ for all $x$ in $\|x\| \leqq \delta$ and all $t \geqq 0$.

We shall assume that every function $V(x, t)$ we will consider possesses continuous first order partial derivatives for all $(x, t)$ in $\Re$. We shall then say that " $d V / d t \leqq 0$ by virtue of (1)" if $\partial V / \partial t+\operatorname{grad} V(x, t) \cdot p(x, t) \leqq 0$ for all $(x, t)$ in $\Re$. Similarly, we shall say that " $d V / d t$ is negative definite by virtue of (1)" if there exists a positive definite function $W(x)$ such that $\partial V / \partial t+\operatorname{grad} V(x, t)$. $p(x, t) \leqq-W(x)$ for all $(x, t)$ in $\Re$. A positive definite function $V(x, t)$ which admits an infinitely small upper bound and for which $d V / d t$ is negative definite by virtue of (1) is commonly called a Liapunov function for the system (1). 
We now state the following two theorems on stability which were first established by Liapunov [9].

Theorem I. If there exists a positive definite function $V(x, t)$ such that $d V / d t \leqq 0$ by virtue of (1), then $x(t) \equiv 0$ is stable.

Theorem II. If there exists a positive definite function $V(x, t)$ admitting an infinitely small upper bound such that $d V / d t$ is negative definite by virtue of (1), then $x(t) \equiv 0$ is asymptotically stable.

Let us observe that in theorem II the hypothesis on the existence of a Liapunov function is quite restrictive. The severity of this restriction can best be seen from the following lemma which strengthens an earlier result of $K$. Persidskii [22].

Lemma. If the system (1) possesses a Liapunov function, then given any $t_{0} \geqq 0$, any sufficiently small $x_{0}$, and any positive $M<1$, there exists a $T\left(M, x_{0}\right)>0$ such that $\left\|x\left(t_{0}+t, x_{0}, t_{0}\right)\right\| \leqq M\left\|x_{0}\right\|$ for all $t \geqq T$.

Proof. We first make the following observations: (a) By hypothesis there exist positive definite functions $V(x, t), W_{1}(x), W_{2}(x)$ such that along any solution $x\left(t, x_{0}, t_{0}\right)$ of (1) with sufficiently small $x_{0}$ we have

$$
\begin{gathered}
V(x, t) \geqq W_{1}(x) \\
\frac{d V(x, t)}{d t}=\frac{\partial V(x, t)}{\partial t}+\operatorname{grad} V(x, t) \cdot p(x, t) \leqq-W_{2}(x)
\end{gathered}
$$

and hence

$$
0<W_{1}(x) \leqq V(x, t)=V\left(x_{0}, t_{0}\right)+\int_{t_{0}}^{t} \frac{d V}{d t} d t
$$

(b) Since $W_{1}(x)$ is positive definite, there exists a constant $L^{*}=L^{*}\left(M, x_{0}\right)$ such that $W_{1}(x) \leqq L^{*}$ implies $\|x\| \leqq M\left\|x_{0}\right\|$.

(c) Consider the sequence of constants $M^{i}\left\|x_{0}\right\|, i=0,1,2, \cdots$. Since $V(x, t)$ admits an infinitely small upper bound, sup $V(x, t)$ for $\|x\|=M^{i}\left\|x_{0}\right\|, t \geqq 0$ exists; let it be designated by $L_{i}$. We have, moreover, $L_{i} \rightarrow 0$ as $i \rightarrow \infty$, and hence there is a smallest index $k=k\left(M, x_{0}\right)$ such that $L_{k} \leqq L^{*}$.

We turn now to the proof proper. Since $M<1$, and $x\left(t_{0}, x_{0}, t_{0}\right)=x_{0}$ we have $\left\|x\left(t, x_{0}, t_{0}\right)\right\|>M\left\|x_{0}\right\|$ for $\left|t-t_{0}\right|$ sufficiently small. Let $l_{0}=l_{0}\left(M, x_{0}\right)$ be a lower bound of $W_{2}(x)$ for $\|x\|>M\left\|x_{0}\right\|$. Then, so long as $\left\|x\left(t, x_{0}, t_{0}\right)\right\|>$ $M\left\|x_{0}\right\|$, we find from (3), (c),

$$
0<W_{1}(x) \leqq L_{0}-l_{0}\left(t-t_{0}\right)
$$

Therefore there must exist a first time $t_{1}$ satisfying $t_{0}<t_{1}<t_{0}+L_{0} / l_{0}$ such that $\left\|x\left(t_{1}, x_{0}, t_{0}\right)\right\|=M\left\|x_{0}\right\|$. Now set $x_{1}=x\left(t_{1}, x_{0}, t_{0}\right)$ and consider $x\left(t, x_{1}, t_{1}\right)$ for $t \geqq t_{1}$. [We note that by the uniqueness of solutions, $x\left(t, x_{1}, t_{1}\right), t \geqq t_{1}$, is the same trajectory as $x\left(t, x_{0}, t_{0}\right), t \geqq t_{1}$.] Let $l_{1}=l_{1}\left(M, x_{0}\right)$ be a lower bound of $W_{2}(x)$ for $\|x\|>M\left\|x_{1}\right\|=M^{2}\left\|x_{0}\right\|$. Then, so long as $\left\|x\left(t, x_{1}, t_{1}\right)\right\|>M\left\|x_{1}\right\|$, we shall have by (3) and (c), 


$$
0<W_{1}(x) \leqq L_{1}-l_{1}\left(t-t_{1}\right) .
$$

Therefore there must exist a first time $t_{2}$ satisfying $t_{1}<t_{2}<t_{1}+L_{1} / l_{1}$ such that $\left\|x\left(t_{2}, x_{1}, t_{1}\right)\right\|=M\left\|x_{1}\right\|=M^{2}\left\|x_{0}\right\|$. We set $x_{2}=x\left(t_{2}, x_{1}, t_{1}\right)$.

Continuing in this way, we can define a sequence of times $t_{1}, t_{2}, \cdots, t_{n}, \cdots$ and of vectors $x_{1}, x_{2}, \cdots, x_{n}, \cdots$ with $x_{n}=x\left(t_{n}, x_{n-1}, t_{n-1}\right)$ such that $\left\|x_{n}\right\|=$ $\left\|x\left(t_{n}, x_{n-1}, t_{n-1}\right)\right\|=M\left\|x_{n-1}\right\|=M^{n}\left\|x_{0}\right\|$. Let the integer $k$ be selected as in (c). We have by (3) for all $t \geqq t_{k}$,

$$
0<W_{1}(x) \leqq V(x, t)=V\left(x_{k}, t_{k}\right)+\int_{t_{k}}^{t} \frac{d V}{d t} d t \leqq V\left(x_{k}, t_{k}\right),
$$

so that by $(c), W_{1}(x) \leqq L_{k} \leqq L^{*}$. Since by $(b), W_{1}(x) \leqq L^{*}$ implies $\|x\| \leqq$ $M\left\|x_{0}\right\|$, we have $\left\|x\left(t, t_{k}, x_{k}\right)\right\| \leqq M\left\|x_{0}\right\|$ for all $t \geqq t_{k}$. Now observe that $t_{n-1}<t_{n}<t_{n-1}+L_{n-1} / l_{n-1}$. Hence $t_{0}<t_{k}<t_{0}+\sum_{i=0}^{k-1} L_{i} / l_{i}$ where this last sum depends merely upon $M$ and $x_{0}$, but is independent of $t_{0}$. Consequently, if we set $T\left(M, x_{0}\right)=\sum_{i=0}^{k-1} L_{i} / l_{i}$, then $a$ fortiori $\left\|x\left(t, x_{0}, t_{0}\right)\right\| \leqq M\left\|x_{0}\right\|$ for all $t \geqq t_{0}+T$, or, $\left\|x\left(t_{0}+t, x_{0}, t_{0}\right)\right\| \leqq M\left\|x_{0}\right\|$ for all $t \geqq T$. This completes the proof.

Liapunov's theorems on stability were improved by Malkin [15], Khalikov $[5,6]$ and Marachkov [17], the latter's extension being the most important one. For Marachkov proved asymptotic stability for systems of form (1) with bounded right hand side without requiring the positive definite function $V(x, t)$ to admit an infinitely small upper bound. Converse theorems were proved for linear systems by Malkin [10, 11, 12] and for more general systems by Massera [18]; his assumptions for linear systems imply a weaker type of stability than that on which Malkin's results are based, as we shall state below.

3. Linear systems. Let us now consider the linear system

$$
\frac{d x}{d t}=A(t) x
$$

where $A(t)$ is a continuous and uniformly bounded matrix function of $t$ for all $t \geqq 0$. Let $x_{1}(t), x_{2}(t), \cdots, x_{n}(t)$ be $n$ linearly independent solutions of (5), which we conveniently combine into the matrix $X(t)$; thus $|X(t)| \neq 0$ for all $t \geqq 0$ so that $X^{-1}(t)$ exists. Then the solution of (5) which reduces to $x_{0}$ when $t=t_{0}$ is given by

$$
x\left(t, x_{0}, t_{0}\right)=X(t) X^{-1}\left(t_{0}\right) x_{0} .
$$

Observe that the matrix function $X(t) X^{-1}\left(t_{0}\right)$ is independent of the particular set of solutions $x_{1}(t), x_{2}(t), \cdots, x_{n}(t)$. For if this set is replaced by another, the matrix $X(t)$ is replaced by $Y(t)=X(t) P$ where $P$ is a non-singular constant matrix; hence $Y(t) Y^{-1}\left(t_{0}\right)=X(t) X^{-1}\left(t_{0}\right)$.

We now state our main theorem. 
Theorem 1. A necessary and sufficient condition for any set of $n$ linearly independent solutions $x_{1}(t), x_{2}(t), \cdots, x_{n}(t)$ of $(5)$ to satisfy for all $t \geqq t_{0} \geqq 0$

$$
\left\|X(t) X^{-1}\left(t_{0}\right)\right\| \leqq M \exp \left[-\alpha\left(t-t_{0}\right)\right]^{*},
$$

where $X(t)=\left\{x_{j}(t)\right\}$ and $M$ and $\alpha$ are positive constants independent of $t_{0}$, is that (5) possesses a Liapunov function $V(x, t)$ which is a quadratic form in $x$ with coefficients that are continuously differentiable and uniformly bounded functions of $t$ for all $t \geqq 0$.

To prove the sufficiency part we need to show that under the hypothesis every solution $x\left(t, x_{0}, t_{0}\right)$ of (5) satisfies for all $t \geqq t_{0} \geqq 0$

$$
\left\|x\left(t, x_{0}, t_{0}\right)\right\| \leqq N \exp \left[-\alpha\left(t-t_{0}\right)\right]
$$

where $N$ and $\alpha$ are positive constants independent of $t_{0}$. For if (8) is satisfied for every solution, we see from (6) by letting $x_{0}=I_{j}, j=1,2, \cdots, n$, where $I_{j}$ is the $j^{\text {th }}$ column of the identity matrix I, that

$$
\left\|X(t) X^{-1}\left(t_{0}\right)\right\| \leqq \sum\left\|\left[X(t) X^{-1}\left(t_{0}\right)\right]_{j}\right\| \leqq n N \exp \left[-\alpha\left(t-t_{0}\right)\right]
$$

which implies (7) with $M \geqq n N$.

Put $y\left(t, x_{0}, t_{0}\right)=\exp \left[k\left(t-t_{0}\right)\right] x\left(t, x_{0}, t_{0}\right)$ so that $y\left(t, x_{0}, t_{0}\right)$ is the solution of the system

$$
\frac{d y}{d t}=(A(t)+k I) y
$$

which satisfies $y\left(t_{0}, x_{0}, t_{0}\right)=x_{0}$. By hypothesis there exist positive definite quadratic forms $W_{0}(x), W_{1}(x), W_{2}(x)$ such that the Liapunov function $V(x, t)$ satisfies

$$
\begin{gathered}
W_{0}(x) \leqq V(x, t) \leqq W_{1}(x) \\
\frac{\partial V(x, t)}{\partial t}+\operatorname{grad} V(x, t) \cdot A(t) x \leqq-W_{2}(x)
\end{gathered}
$$

for all $x$ and all $t \geqq 0$. Hence along the solution $y\left(t, x_{0}, t_{0}\right)$ of (10) we find, using the hypothesis that $V(x, t)$ is a quadratic form in $x$,

$$
\begin{aligned}
\frac{d V(y, t)}{d t}=\frac{\partial V(y, t)}{\partial t}+ & \operatorname{grad} V(y, t) \cdot[A(t)+k I] y \\
& \leqq-W_{2}(y)+2 k V(y, t) \leqq-\left[W_{2}(y)-2 k W_{1}(y)\right] .
\end{aligned}
$$

By taking $k>0$ sufficiently small it is evident that we can make the quadratic form $W_{3}(y)=W_{2}(y)-2 k W_{1}(y)$ positive definite so that

* Here we use for the matrix $X=\left(x_{i j}\right)$ the norm $\|X\|=\left(\Sigma x_{i i^{2}}\right)^{3}$. 


$$
\frac{d V(y, t)}{d t} \leqq-W_{3}(y)
$$

is negative definite. Hence we have along the solution $y\left(t, x_{0}, t_{0}\right)$

$$
V(y, t) \leqq V\left(x_{0}, t_{0}\right) \leqq W_{1}\left(x_{0}\right),
$$

from which we conclude that $\left\|y\left(t, x_{0}, t_{0}\right)\right\| \leqq N$ for all $t \geqq t_{0} \geqq 0$ where $N$ is a positive constant independent of $t_{0}{ }^{*}$ It follows that

$$
\left\|x\left(t, x_{0}, t_{0}\right)\right\| \leqq N \exp \left(-k\left(t-t_{0}\right)\right)
$$

for all $t \geqq t_{0} \geqq 0$, and so (8) will be satisfied for every positive $\alpha \leqq k$.

In the proof of the necessity part of the theorem we make use of a recent result of Malkin's [16] which we reproduce here in full for the special case of quadratic forms.

Let $P$ be a (constant) positive definite symmetric matrix and $X(t)$ the matrix of any set of $n$ linearly independent solutions of (5); define the matrix $Y(\tau, t)=$ $X(\tau) X^{-1}(t)$. Then $x^{\prime} Y^{\prime}(\tau, t) P Y(\tau, t) x$, where the dash denotes transposition, is a positive definite quadratic form in $x$ for all $\tau, t \geqq 0$.

Consider now the quadratic form in $x$

$$
V(x, t)=\int_{t}^{\infty} x^{\prime} Y^{\prime}(\tau, t) P Y(\tau, t) x d \tau
$$

From the hypothesis (7) we find

$$
\begin{aligned}
|V(x, t)| & \leqq \int_{t}^{\infty}\left\|x^{\prime}\right\|\left\|Y^{\prime}(\tau, t)\right\|\|P\|\|Y(\tau, t)\|\|x\| d \tau \\
& \leqq\|x\|^{2} M^{2}\|P\| \int_{t}^{\infty} e^{-2 \alpha(\tau-t)} d \tau=\frac{1}{2 \alpha}\|x\|^{2} M^{2}\|P\|,
\end{aligned}
$$

so that the coefficients of $V(x, t)$ are uniformly bounded functions of $t$ for all $t \geqq 0$. We assert that $V(x, t)$ is positive definite.

Write $Z(\tau, t)=|Y(\tau, t)| Y^{-1}(\tau, t)$, where

$$
|Y(\tau, t)|=\left|X(\tau) X^{-1}(t)\right|=\exp \left[\int_{t}^{\tau} \operatorname{trace} A(s) d s\right] .
$$

Since $Z(\tau, t)$ is the adjoint of $Y(\tau, t)$, we have in view of $(7)\|Z(\tau, t)\| \leqq N$ for all $\tau \geqq t \geqq 0$ and therefore for all $y$ and all $\tau \geqq t \geqq 0$ and any constant $\lambda>0$

$$
y^{\prime} P y-\lambda y^{\prime} Z^{\prime}(\tau, t) Z(\tau, t) y \geqq y^{\prime} P y-\lambda N^{2}\|y\|^{2}=y^{\prime}\left(P-\lambda N^{2} I\right) y .
$$

* It is clear that we do not need $W_{3}(y)$ positive definite but merely $W_{3}(y) \geqq 0$ for all $y$. This would increase the upper bound of admissible values of $k>0$ and hence of $\alpha$. If $W_{3}(y)$ is positive definite, then $y\left(t, x_{0}, t_{0}\right) \rightarrow 0$ as $t \rightarrow \infty$ by LIAPUnov's theorem II. 
$P$ being positive definite, we can choose $\lambda_{0}>0$ so small that $P-\lambda_{0} N^{2} I$ is also positive definite. Hence, letting $y=Y(\tau, t) x$ in the left hand side of (19), we will have for $\lambda=\lambda_{0}$ and all $\tau \geqq t \geqq 0$

$$
\begin{aligned}
x^{\prime} Y^{\prime}(\tau, t) P Y(\tau, t) x & -\lambda_{0} x^{\prime} Y^{\prime}(\tau, t) Z^{\prime}(\tau, t) Z(\tau, t) Y(\tau, t) x \\
= & x^{\prime} Y^{\prime}(\tau, t) P Y(\tau, t) x-\lambda_{0}|Y(\tau, t)|^{2}\|x\|^{2}>0,
\end{aligned}
$$

and thus

$$
V(x, t)>\lambda_{0}\|x\|^{2} \int_{t}^{\infty}|Y(\tau, t)|^{2} d \tau
$$

Now observe that by virtue of (7) we can conclude that $|Y(\tau, t)| \rightarrow 0$ as $\tau \rightarrow$ $\infty$ for any (fixed) $t \geqq 0$. Hence we find, using (18),

$$
\begin{aligned}
\int_{t}^{\infty} \operatorname{trace} A(\tau) \exp \left(2 \int_{t}^{\tau} \operatorname{trace} A(s) d s\right) d \tau & =-\frac{1}{2} \\
= & \operatorname{trace} A(\xi) \int_{t}^{\infty}|Y(\tau, t)|^{2} d \tau,
\end{aligned}
$$

where the last equality was obtained by applying the mean value theorem to the left hand integral; the undetermined constant $\xi$ lies within the interval $t \leqq \xi<\infty$. Since trace $A(\xi)$ is bounded for all $\xi \geqq 0$ by assumption, we conclude that $\int_{t}^{\infty}|Y(\tau, t)|^{2} d \tau$ cannot become zero as $t \rightarrow \infty$; and since the integral is positive, it must therefore have a positive lower bound $\mu$. Hence we have from (20)

$$
V(x, t) \geqq \lambda_{0} \mu\|x\|^{2},
$$

i.e. $V(x, t)$ is positive definite.

Along any solution $x\left(t, x_{0}, t_{0}\right)$ of $(5)$ we find by differentiating (16) that

$$
\frac{d V(x, t)}{d t}=-x^{\prime} P x
$$

Thus, $P$ being a positive definite symmetric matrix, $d V(x, t) / d t$ is negative definite by virtue of (5), and so $V(x, t)$ is a Liapunov function which is a quadratic form in $x$ with coefficients that are uniformly bounded functions of $t$ for all $t \geqq 0$. The proof is now complete.

Theorem 1 could have been stated equally well in terms of the general solution $x\left(t, x_{0}, t_{0}\right)$ of $(5)$; i.e. (7) could have been replaced by the statement that every solution $x\left(t, x_{0}, t_{0}\right)$ satisfy for all $t \geqq t_{0} \geqq 0$.

$$
\left\|x\left(t, x_{0}, t_{0}\right)\right\| \leqq N \exp \left[-\alpha\left(t-t_{0}\right)\right]
$$

where $N$ and $\alpha$ are positive constants independent of $t_{0}$. For as we have seen (24) implies (7), and it is clear that (7) implies (24). As stated, theorem 1, how- 
ever, brings out best the connection between the existence of a Liapunov function and the classical "variation of parameters" technique.

Massera has shown [18] that if $x(t) \equiv 0$ is an asymptotically stable solution of (5) then there exists a Liapunov function for the system (5), thereby establishing the necessity of Liapunov's theorem II for linear systems. However. Massera's construction yields a Liapunov function that is not a quadratic form in $x$ and does not admit an infinitely small upper bound but instead satisfies somewhat milder conditions. (This is, of course, to be expected in view of theorem 1.)

It is evident that under the conditions of theorem 1 the characteristic numbers of a set of $n$ linearly independent solutions of (5), as defined by Liapunov [9], are all positive. As was shown by Malkin [12] we cannot, however, conclude therefrom that the system (5) is regular in Liapunov's sense [9].

4. Non-homogeneous linear and non-linear systems. Theorem 1 sheds new light on classical results of Perron $[19,20]$ concerning non-homogeneous linear systems

$$
\frac{d y}{d t}=A(t) y+b(t)
$$

where $b(t)$ is a continuous vector function of $t$ for all $t \geqq 0$, and also on more recent results for non-linear systems of the form

$$
\frac{d y}{d t}=A(t) y+q(y, t)
$$

where $q(y, t)$ satisfies certain rather mild requirements to be specified later. Perron was the first to establish necessary and sufficient conditions so that all solutions of (25) are bounded (i) for every bounded vector function $b(t)$ and (ii) for every vector function $b(t)$ for which $\int^{\infty}\|b(t)\| d t<\infty,[\mathbf{2}, \mathbf{3}, \mathbf{4}, \mathbf{7}, \mathbf{2 0}$. Malkin [12] demonstrated that for $(i)$ these conditions are equivalent to the existence of a Liapunov function for the corresponding homogeneous system. Combining this result with theorem 1 we obtain a formulation which is of much interest in applied work.

Theorem 2. A necessary and sufficient condition for every solution $y\left(t, y_{0}, t_{0}\right)$ of (25) to be bounded for every continuous vector function $b(t)$ that is bounded for all $t \geqq 0$ is that

(a) any set of $n$ linearly independent solutions $x_{1}(t), x_{2}(t), \cdots, x_{n}(t)$ of (5) satisfy for all $t \geqq t_{0} \geqq 0$

$$
\left\|X(t) X^{-1}\left(t_{0}\right)\right\| \leqq M \exp \left[-\alpha\left(t-t_{0}\right)\right]
$$

where $X(t)=\left\{x_{j}(t)\right\}$ and $M$ and $\alpha$ are positive constants independent of $t_{0} ;$ or

(b) there exist a Liapunov function for the system (5) which is a quadratic form in $x$ with coefficients that are uniformly bounded functions of $t$ for all $t \geqq 0$. 
It is easily seen that if either of the hypothesis is satisfied then every solution of (25) is bounded also for every continuous vector function $b(t)$ for which $\int^{\infty}\|b(t)\| d t<\infty,[4]$.

Concerning the non-linear system (26) we can now state similar results, for it is well known that the boundedness of every solution of (26) is essentially determined by the behavior of every solution of (25). In fact, we have the following theorem essentially due to Levinson [8].

Theorem 3. Every solution $y\left(t, y_{0}, t_{0}\right)$ of the system

$$
\frac{d y}{d t}=A(t) y+q\left(y, \frac{d y}{d t}, t\right)
$$

with sufficiently small $y\left(t_{0}\right), \dot{y}\left(t_{0}\right)$ will satisfy for all $t \geqq t_{0} \geqq 0$

$$
\left\|y\left(t, y_{0}, t_{0}\right)\right\| \leqq M \exp \left[-\alpha\left(t-t_{0}\right)\right]
$$

where $M$ and $\alpha$ are positive constants independent of $t_{0}$, if $q(y, z, t)$ is continuous for all $y, z$ and all $t \geqq 0, q(0,0, t) \equiv 0$, and satisfies for sufficiently small $\|y\|$ and $\|z\|$ and for all $t \geqq 0$

$$
\|q(y, z, t)\| \leqq \lambda_{1}\|y\|+\lambda_{2}\|z\|
$$

where $\lambda_{1}$ and $\lambda_{2}$ are positive constants dependent upon the upper bound of the matrix $A(t)$ for all $t \geqq 0$, and if

(a) any set of $n$ linearly independent solutions $x_{1}(t), x_{2}(t), \cdots, x_{n}(t)$ of (5) satisfies for all $t \geqq t_{0} \geqq 0$

$$
\left\|X(t) X^{-1}\left(t_{0}\right)\right\| \leqq M \exp \left[-\alpha\left(t-t_{0}\right)\right]
$$

where $M$ and $\alpha$ are positive constants independent of $t_{0}$; or

(b) there exists a Liapunov function for the system (5) which is a quadratic form in $x$ with coefficients that are uniformly bounded functions of $t$ for all $t \geqq 0$.

In Levinson's original formulation the matrix $A(t)$ was assumed constant with all characteristic roots having negative real parts. The theorem as stated above contains a number of related older results as special cases; we mention only Perron [19, 20], Malkin [13], Persidskii [22], Bellman [2] and refer to [4] for further references.

5. Some practical tests. Through theorem 1 the hypothesis in the above stated theorems become rather easy to test, for in many cases a Liapunov function can be constructed explicitly either by mere inspection of the linear system or by application of some known criteria [1, 24, 25]. These criteria are based upon properties of the coefficients of the matrix $A(t)$ of $(5)$ which are generally readily testable. (When $A(t)$ is constant, the requirement for the existence of a Liapunov function obviously is that the characteristic roots of $A$ all have negative real parts.) For linear equations of the second order, 


$$
\ddot{x}+p(t) \dot{x}+q(t) x=0,
$$

where $0<l \leqq p(t) \leqq L, 0<m \leqq q(t) \leqq M$, Starzhinskii has shown that a Liapunov function that is a quadratic form in $x$ and $\dot{x}$ will exist if the inequalities

$$
\begin{gathered}
l>\sqrt{M}-\sqrt{m} \\
M<\frac{M+2 \sqrt{m M}+5 m}{\sqrt{M}-\sqrt{m}}
\end{gathered}
$$

are satisfied. A similar set of inequalities can be derived for linear equations of the third order, [25]. 


\section{REFERENCES}

[1] Antosiewicz, H. A. "A note on asymptotic stability." Quart. Appl. Math., Vol. 9(1951), 317-319.

[2] Bellman, R. "On the boundedness of solutions of non-linear differential and difference equations." Trans. Amer. Math. Soc. vol. 46(1948), 374-388.

[3] _ "On an application of a Banach-Steinhaus theorem to the study of the boundedness of solutions of non-linear differential and difference equations." Ann. of Math., vol. 49(1948), 515-522.

[4] - "A survey of the theory of the boundedness etc." Office of Naval Research, 1949.

[5] Khalizov, N. S. "On the stability in the large of the solutions of differential equations," Izvestiya Kazan. Fiz-Mat. Obschchestva, vol. 9(1937), 31-57.

[6] — "Remark on a theorem on the stability of movement," Ibid., vol. 11(1938), 199.

[7] Levinson, N. "The asymptotic behavior of a system of linear differential equations." Amer. Jour. Math., vol. 68 (1946), 1-6.

[8] _ "On stability of non-linear systems of differential equations." Colloquium Math., vol. 2 (1949), 40-45.

[9] Liapounoff, M. A. "Problème général de la stabilité du mouvement." Ann. of Math. Studies, No. 17, Princeton Univ. Press, 1947.

[10] MaLkin, I. G. "Das Existenzproblem von Liapounoff'schen Funktionens." Izvestiya Kazan. Fiz-Mat. Obschchestva, vol. 4 (1929-30), 51-62.

[11] _ "On the problem of the existence of a function of Liapounoff." Ibid., vol. 5 (1931), 63-84.

[12] “Certain questions on the theory of the stability of motion in the sense of Liapounoff." Amer. Math. Soc. Translation No. 20, 1950.

[13] —U "Über die Bewegungsstabilität nach der ersten Näherung." C. R. (Doklady) Acad. Sci. URSS, vol. 18 (1938), 159-162.

[14] — "Verallgemeinerung des Fundamentalsatzes von Liapounoff über die Stabilität der Bewegungen." Ibid. vol. 18 (1938), 162-164.

[15] — "On stability under constantly acting disturbances." Amer. Math. Soc. Translation No. 8, 1950.

[16] — "On the construction of Liapunov functions for systems of linear equations." Akad. Nauk SSSR. Prikl. Mat. Meh., vol. 16 (1952), 239-242.

[17] Marachrov, M. "On a theorem on stability." Izvestiya Kazan. Fiz-Mat. Obschchestva, vol. 12 (1940), 171-174.

[18] MASSera, J. L. "On Liapounoff's conditions of stability." Ann. of Math., vol. 50 (1949), 705-721.

[19] Perron, O. "Über Stabilität und asymptotisches Verhalten der Integrale von Differentialgleichungssystemen." Math. Zeit. vol. 29 (1928), 129-160.

[20] —Die Stabilitätsfrage bei Differentialgleichungen." Math. Z., Vol. 32 (1930), 703-728.

[21] Persidskir, K. "On a theorem on the stability of movement," Izvestiya Kazan. Fiz-Mat. Obschchestva, vol. 6 (1932-33), 76-79.

[22] —Über die Stabilität einer Bewegung nach der ersten Näherung." Mat. Sbornik, vol. 40 (1933), 284-293.

[23] — "On the theory of stability of integrals of systems of differential equations." Izvestiya Fiz-Mat. Obschchestva Kazan. Univ. (Ser. 3), vol. 8 (1936-37), 47-85 and vol. 11 (1938), 29-45.

[24] Starzhinskir, V. M. "Sufficient conditions for stability of a mechanical system with one degree of freedom." Akad. Nauk SSSR. Prikl. Mat. Meh., vol. 16 (1952), 369-374.

[25] — "On stability of perturbed motions in a particular case." Ibid., vol. 16 (1952), 500-504.

[26] Truitzinsky, W. J. "Properties of growth for solutions differential equations of dynamical type." Trans. Amer. Math. Soc., vol. 50 (1941), 252-294. 